This item was submitted to Loughborough's Institutional Repository (https://dspace.lboro.ac.uk/) by the author and is made available under the following Creative Commons Licence conditions.

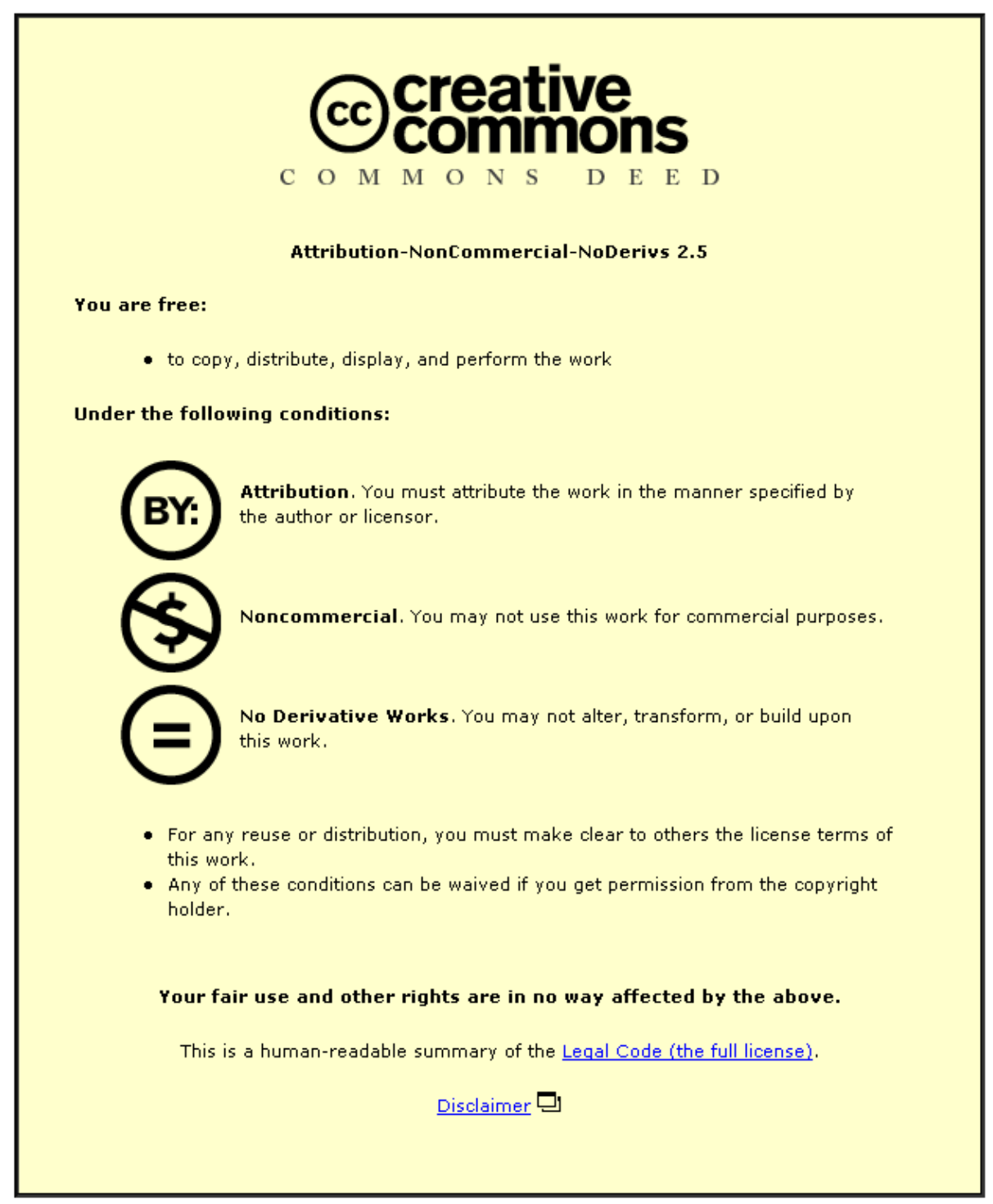

For the full text of this licence, please go to: http://creativecommons.org/licenses/by-nc-nd/2.5/ 
Journal of Applied Biomechanics, 17, 326-334, 2001

\title{
High resolution determination of body segment inertial parameters and their variation due to soft tissue motion.
}

\author{
Matthew T. G. Pain ${ }^{1}$ and John H. Challis ${ }^{2}$ \\ ${ }^{1}$ Dept. of Physical Education, Sports Science \& Recreation Management Loughborough University LE11 3TU, UK \\ ${ }^{2}$ Biomechanics Lab, 39 Rec Bldg, Dept of Kinesiology, Penn State Uni, University Park PA 16802-3408, USA
}

\begin{abstract}
There were two purposes of this study: to evaluate a new method for measuring segmental dimensions for determining body segment inertial parameters (BSIP), and to evaluate the changes in mass distribution within a limb as a consequence of muscular contraction. BSIP were calculated by obtaining surface data points of the body under investigation using a sonic digitizer, interpolating them into a regular grid and then using Green's theorem which relates surface to volume integrals. Four skilled operators measured a test object, the error was approximately $2.5 \%$ and repeatability $1.4 \%$ (coefficient of variation) in the determination of BSIP. Six operators took repeat measures on human shanks, coefficients of variation were typically around 5\%, and 3\% for the more skilled operators. Location of the center of mass of the shank was found to move up $1.7 \mathrm{~cm}$ proximally when the triceps surae muscles went from a relaxed state to causing plantar flexion. The force during an impact associated with such motion of the soft tissue of the shank was estimated to be up to $300 \mathrm{~N}$. In summary a new repeatable and accurate method for determining BSIP has been developed, and has been used to evaluate body segment mass redistribution due to muscular contraction.
\end{abstract}

KEYWORDS: segmental inertias, wobbling mass

\section{INTRODUCTION}

For the mechanical analysis of human movement human body segments are frequently assumed to be rigid bodies, with the inertial parameters of these bodies required for detailed analyses. Body segment inertial parameters (BSIP) can be estimated in a number of different ways including the use of statistical models (e.g. Hinrichs, 1985), medical imaging techniques (e.g. Mungiole \& Martin, 1990), and modeling the body segments as series of geometric solids (e.g. Hatze, 1980). The geometric modeling techniques represent the segments of the body using geometric shapes. The dimensions of these shapes are obtained by taking anthropometric measurements on the experimental subject (e.g. segment length and perimeter). From these data it is possible to obtain an approximation of segment volume, then by providing an estimate of the segment density it is possible to estimate the inertial parameters.

When determining body segment inertial parameters via the geometric solid modeling technique there are a number of different ways in which the measurements can be taken. For example Yeadon (1990) and Hatze (1980) both made their measurements directly on the subject, Jensen (1978) took the measurements manually from a photographic image of the subject, while Sarfaty \& Ladin (1993) and Baca (1996) presented automated video based techniques for determining the appropriate dimensions. All these solid modeling techniques transform measurements from a subject to analogous dimensions on a regular geometric shape, that has analytical solutions which permit determination of its inertial parameters. This allows effective calculation of the inertial parameters only when the geometry of the segment can be approximated effectively by these geometrical shapes

Crisco \& McGovern (1998) showed how Green’s theorem could be used to evaluate inertial parameters of solid objects by simulating digitized cross sections in MATLAB. Green's theorem relates boundary integrals to area integrals; the theorem can be found in mathematical texts (e.g. Stephenson, 1973). The dependence of the error, between the true inertial parameters and those 
calculated using the discrete versions of Green's theorem, on symmetry and number of cross-sections was examined. They found asymmetry of an object and fewer cross-sections gave the greater errors but if a sufficient number of slices were taken the errors could be reduced to near zero. For their computer generated data they found that this method was both accurate and computationally efficient. This method is superior to the geometric solid methods as the inertial parameters are calculated for the actual shape of the object not a geometric simulacrum. Tests on real objects were not carried out.

Human body segments are not truly rigid and this has a number of influences on the mechanics of human movement. This lack of rigidity can also influence the repeatability with which segment dimensions can be measured. The actual non-rigid motion can also potentially influence the resultant joint forces and moments (Gruber et al., 1998).

There were two purposes of this study. One was to evaluate a new method for measuring segmental dimensions for determining BSIP. The other was to use this technique to evaluate the changes in mass distribution within a limb as a consequence of muscular contraction.

\section{METHODS}

To determine BSIP using Green's theorem sets of coordinates describing the cross-sections need to be collected from the surface of the object under investigation. To measure and obtain uniformly spaced cross-sections from an object would be incredibly time consuming, and would require the object to be marked with a grid. To avoid this the surface was measured at semi random points, while ensuring that the whole surface was covered. These points were then interpolated into a regular grid using a cubic spline. These regular points could then be deconstructed into a series of regularly spaced cross-sections.

A SAC GP-12XL three-dimensional sonic digitizing system (Stratford, CT) was used to record the three-dimensional coordinates of the surfaces of the objects under investigation. The accuracy of the GP-12 was assessed to be $0.4 \mathrm{~mm}$ in the data collection environment. Each object to be measured was placed on a pedestal in the center of the GP-12's triangular receiver array, to minimize line of sight obstructions. The emitter probe was run across the surface, from top to bottom, in lines about 1-2 cm apart, about 25 to 30 lines were traced per object, with the digitizer set on continuous mode. The continuous mode did not operate at a specific frequency but gave approximately two counts per centimeter.

Once the surface points had been recorded, usually around 1200 points, they were loaded into MATLAB (The Math Works Inc., Natick, MA) and visually inspected. Any extreme outliers or erroneous measures were deleted. Software written in MATLAB split the surface points into two halves and reoriented each half so that it could be treated as a surface on an X-Y plane. The $\mathrm{X}$ and $\mathrm{Y}$ coordinates of each surface were interpolated onto a regular grid in the $X-Y$ plane using a cubic spline and a typical grid size of $2.5 \mathrm{~mm}$ by $4 \mathrm{~mm}$. This resulted in $\mathrm{X}-\mathrm{Y}$ and $\mathrm{Z}$ coordinates for a large number of points that reproduced the surface of the object in a regular pattern (figure 1). The Z axis was aligned with each body's longitudinal axis, and the $\mathrm{X}$ and $\mathrm{Y}$ were transverse axes, the origin of this axis system was at the body's center of mass. 

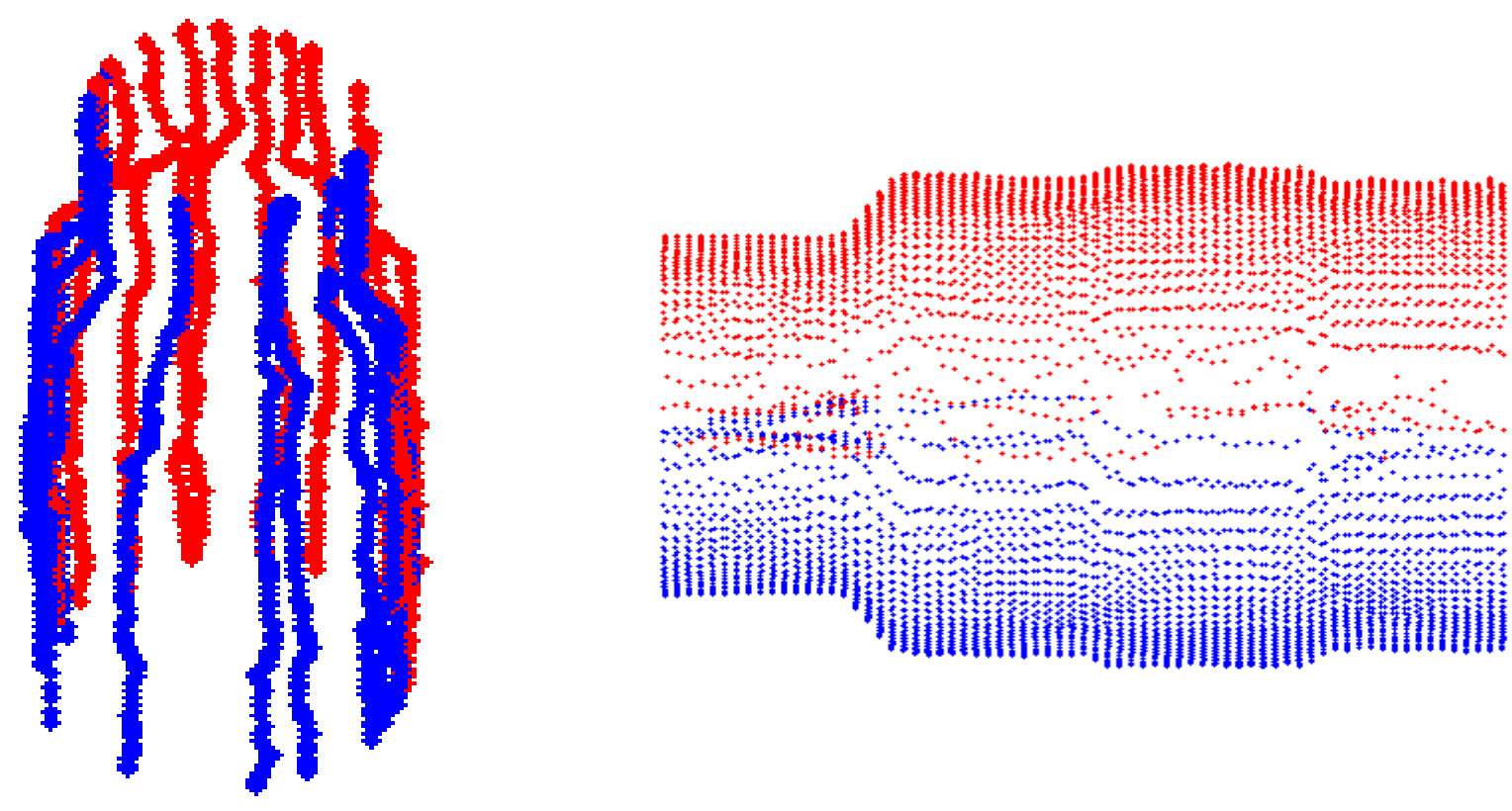

Figure 1 - On the left is an image of the raw data points collected from the test object. On the right is the rotated and interpolated image of the test object.

A second program implemented discrete Green's equations as described by Crisco \& McGovern (1998). Once a set of inertial parameters were obtained the centroid locations were used to iteratively reposition the object so that its centroids lay at $\left[\begin{array}{lll}0 & 0 & 0\end{array}\right]$ to reduce integration errors. Normally five to six steps were necessary for the relocations, and recalculation of inertial values to lead to convergence about the origin. This is necessary, as during boundary integrals a missed point or incomplete integration will produce errors dependant on the unmatched integration area.

There were two parts to the evaluation, one was to evaluate the accuracy of the measurement of the BSIP, and the second was to assess the repeatability with which this could be done. In all cases the operators were trained in the measurement procedure and given precise anatomical definitions of segment boundaries. All subjects who were measured gave informed consent.

To assess accuracy a wooden model was machined to have the same approximate dimensions of a human shank. The model was $0.325 \mathrm{~m}$ long, with a maximum perimeter of $0.386 \mathrm{~m}$, and a minimum perimeter of $0.276 \mathrm{~m}$. Direct measurements were made of its mass, volume, and dimensions. From these the remainder of its inertial parameters were determined assuming uniform density. Four operators made measurements of this model. Data collection of the wooden model typically took under 150 seconds. Accuracy was evaluated by expressing the absolute difference between the estimated and true value as a percentage of the true value.

To assess the repeatability four operators each performed two measures on the model shank. In addition six other operators performed repeat measures on the shanks of various subjects. To assess repeatability the standard deviation of the sets of repeat measures were computed and expressed as a percentage of the mean value (coefficient of variation). Density data for the shanks was taken from Clauser et al. (1969).

Three male subjects (height $1.75 \mathrm{~m} \pm 0.05$, mass $75 \mathrm{~kg} \pm 5.5$, shank length $0.403 \mathrm{~m} \pm 0.015$, maximum perimeter $0.374 \mathrm{~m} \pm 0.028$, minimum perimeter $0.233 \mathrm{~m} \pm 0.007$ ) had the inertial parameters of their shanks estimated under two conditions, by one operator for whom repeatability was assessed. Condition one the knee was flexed to 90 degrees, and the ankle maximally plantar-flexed. Condition two the knee was near full extension and the ankle was in the mid-range of motion. Data collection of a shank typically took under 180 seconds. 


\section{RESULTS}

With practice an operator could collect accurate surface measurements of a test object and these could be used to compute BSIP with an error of approximately $2.5 \%$ (table 1 ). The moments of inertia about the transverse axes (Ixx and Iyy) were the same, but estimated with different accuracies; this was caused by measurement errors. The repeatability of the measurement of the inertial parameters varied for different parameters (table 1), but overall had a mean value around $1.4 \%$.

Repeat measures on the shanks of subjects gave an error in repeatability of around $5 \%$ (table 2). However this value is much lower if the more consistent operators only are considered (operators $\mathrm{D}, \mathrm{E}$, and F).

Table 1 - Accuracy and repeatability of inertial values for measurement of the test object made by four operators. The measure of repeatability is the mean coefficient of variation of measurements made by four operators

\begin{tabular}{|c|c|c|c|c|c|c|}
\hline & $\begin{array}{c}\text { Mass } \\
(\%)\end{array}$ & $\begin{array}{c}\text { Length } \\
(\%)\end{array}$ & $\begin{array}{l}\text { Center of } \\
\text { Mass (\%) }\end{array}$ & $\begin{array}{l}\text { Ixx } \\
(\%)\end{array}$ & $\begin{array}{l}\text { lyy } \\
\text { (\%) }\end{array}$ & $\begin{array}{l}\text { Izz } \\
(\%)\end{array}$ \\
\hline \multicolumn{7}{|l|}{ Accuracy } \\
\hline Mean & 0.9 & 1.0 & 2.8 & 1.6 & 6.3 & 1.8 \\
\hline $\begin{array}{l}\text { Range of } \\
\text { Values }\end{array}$ & $0.1-2.3$ & $0.4-2.4$ & $1.8-3.9$ & $0.4-2.3$ & $2.8-7.7$ & $0.0-5.8$ \\
\hline \multicolumn{7}{|c|}{ Repeatability } \\
\hline $\begin{array}{l}\text { Coefficient } \\
\text { of Variation }\end{array}$ & 0.8 & 0.8 & 1.3 & 1.9 & 1.8 & 1.6 \\
\hline
\end{tabular}

Table 2 - The repeatability of BSIP measures made in six different operators as expressed by one standard deviation as a percentage of the mean

\begin{tabular}{|c|c|c|c|c|c|c|c|}
\hline Operator & $\begin{array}{c}\text { Number } \\
\text { of Repeat } \\
\text { Measures }\end{array}$ & $\begin{array}{c}\text { Mass } \\
\text { (\%) }\end{array}$ & $\begin{array}{c}\text { Length } \\
\mathbf{( \% )}\end{array}$ & $\begin{array}{c}\text { Center of } \\
\text { Mass } \\
\mathbf{( \% )}\end{array}$ & $\begin{array}{c}\text { Ixx } \\
\mathbf{( \% )}\end{array}$ & $\begin{array}{c}\text { Iyy } \\
\mathbf{( \% )}\end{array}$ & $\begin{array}{c}\text { Izz } \\
\mathbf{( \% )}\end{array}$ \\
\hline A & 3 & 8.7 & 1.0 & 14.2 & 14.3 & 11.4 & 16.8 \\
\hline B & 3 & 8.4 & 0.6 & 5.3 & 9.7 & 8.8 & 12.0 \\
\hline C & 5 & 6.2 & 0.6 & 8.1 & 9.9 & 2.0 & 7.3 \\
\hline D & 3 & 1.6 & 0.7 & 1.1 & 10.9 & 4.8 & 2.5 \\
\hline E & 3 & 3.8 & 0.7 & 1.5 & 1.6 & 5.0 & 3.0 \\
\hline F & 2 & 1.1 & 0.9 & 1.1 & 5.0 & 0.3 & 3.4 \\
\hline
\end{tabular}

The mean location of the center of mass in the normal position was $42 \%$ of the length of the shank from the proximal end, which is consistent with values reported in the relevant literature (e.g. Dempster, 1955). The location of the center of mass moved 1.1, 1.7 and $1.3 \mathrm{~cm}$ closer to the proximal end of the shank for the three subjects when their ankle went from a normal to a plantar flexed position. These measures were made by an operator whose repeatability was comparable to the best reported in table 2, therefore these changes were much greater than any error arising due to lack of repeatability in 
the measures. The volume of each segment varied on the same order as the repeatability but changes in maximum dimensions were around $10 \%$. This indicates that as expected segment volume was not changing due to muscle contraction but a redistribution of the muscle was measurable. This helps verify the repeatability of the technique. The moments of inertia increased by $5 \%$ about the longitudinal axis and decreased by $8 \%$ about the two transverse axes. In figure 2 the change in muscle geometry can be clearly seen from the normal to plantar flexed condition. In the plantar flexed condition the two heads of the gastrocnemius are visible and defined closer to the proximal end of the shank.
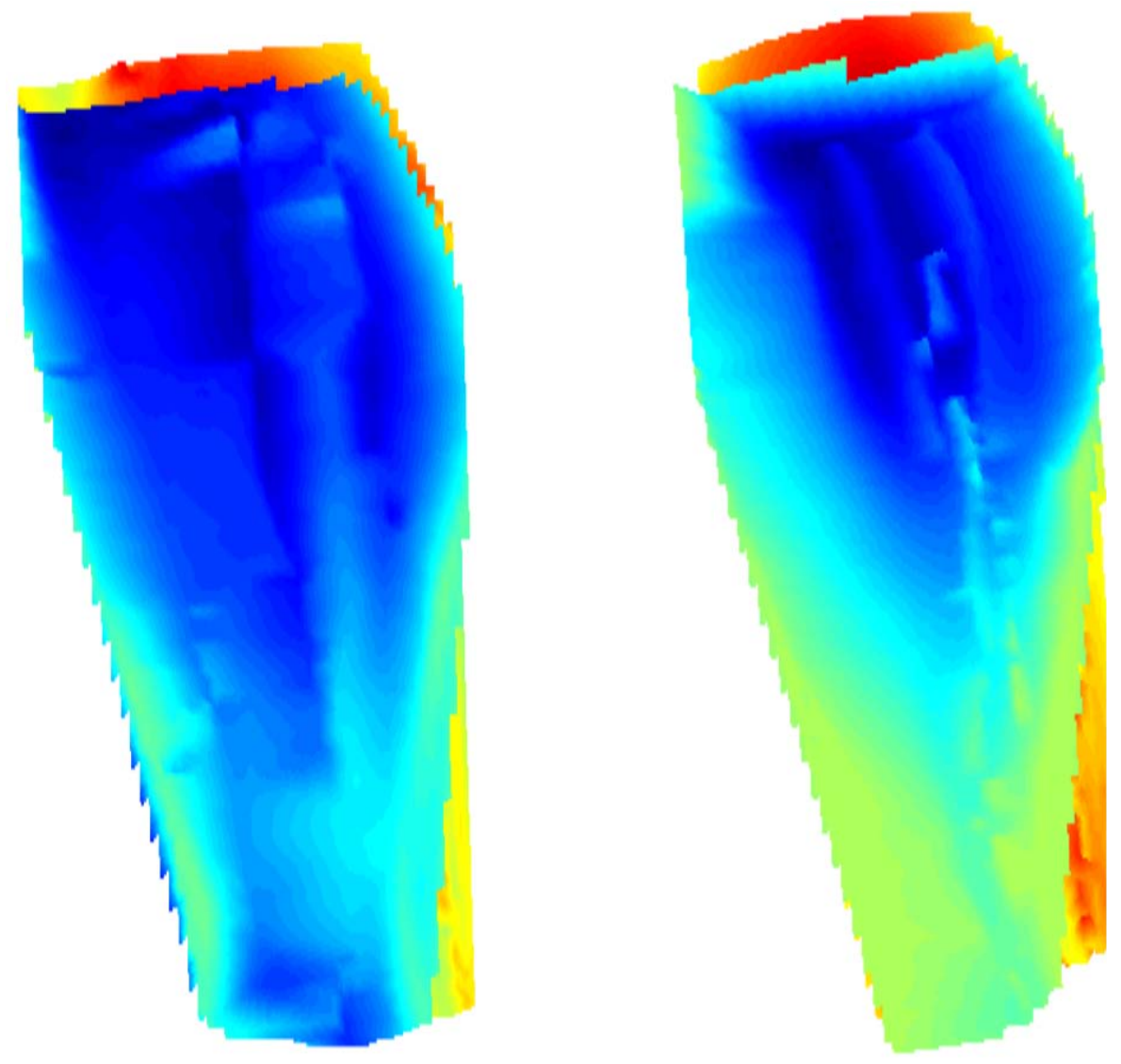

Figure 2 - Surface plots of the shank of one subject in the normal position, left image, and the plantar flexed condition, right image.

\section{DISCUSSION}

A method for determining body segment inertial parameters has been presented. With appropriately trained operators this method can produce accurate and repeatable estimates of body segment inertial parameters. Given this new method is was possible to assess the changes in mass distribution within a limb as a consequence of muscular contraction. Analysis of mass redistribution within the shank showed the inertial parameters did indeed change. These results therefore indicate that during normal movement changes in the segmental inertial parameters can occur, and that when estimating a segments inertial parameters the state of tension in the muscles and the joint angles can influence the determined parameters. This affect was only assessed for the shank, its influence on other body segments is worthy of investigation.

In determining the moments of inertia there was lower accuracy and repeatability about the $\mathrm{Y}$ axis compared with the $\mathrm{X}$ axes, yet for the test objects these moment of inertia should be equal, and 
for the human shanks very similar. In collecting the data it was necessary to interpolate the coordinate data obtained of the objects surface. Due to the nature of the three-dimensional interpolation technique the points at the edges of a surface were not as well defined as the remainder of the points. The lack of accuracy and repeatability in the moment of inertia values about the $\mathrm{Y}$ axis were due to these errors at the edges. This problem could be circumvented by running the programs twice, once as has been done and a second time with the object rotated so the Ixx plane is in error and using the more accurate version of each.

Human limbs are asymmetric and obtaining large numbers of uniformly spaced crosssections can be incredibly time consuming if measurements are to be taken directly from the subject. This can represent the worst case scenario for utilizing Green's theorem as a method of determining BSIP. A method has been presented that provides data that can be successfully used with Green's theorem to determine BSIP. The measurements have a finer resolution than other currently employed techniques. It took about 180 seconds to measure a limb so a whole body could be measured in approximately 45 minutes. This is equivalent to the time reported by Hatze (1980) to take all the measurements required for his model. The detailed shape information provided by this technique may be of use for computer graphic images.

With any measurement made of segment dimensions there are a number of problems. These include tissue depression during measurement, problems of subjects maintaining a required position, and the overall duration of measurement. For the technique it was necessary to specify the density of each segment, in this case the density data of Clauser et al. (1969) was used. The use of any other density data set could not have changed the repeatability of measures, unless segments had been modeled with variable density data along the length of each segment. Unfortunately there is a paucity of variable density data, so it was not possible to examine the influence of this on the estimated inertial parameters. The techniques used here to calculate the inertial parameters allow variable density data along the longitudinal axis to be included simply by specifying a different density for each slice. Variable density along the transverse axis would require digitizing of the different tissues within a slice and could not be easily incorporated. Data from dual photon absorbtiometry could be used to apply variable density data along the longitudinal axis (e.g. Durkin \& Dowling, 1998). When examining the changes in BSIP due to muscular tension the incorporation of different densities for the rigid skeletal structure and the moving soft tissue would be a useful extension of the current work.

The results show that operators measured with both different levels of accuracy and repeatability (tables 1 and 2). These results indicate the importance of properly training operators and assessing their accuracy and repeatability before using their data. On human subjects it is not possible to assess accuracy, but repeatability can be assessed by comparing repeat measures by the same operator. For the operators showing the poorest repeatability in table 2, it would not be feasible to measure changes in segment mass distribution due to movement of the muscle mass. For the more skilled operators the estimation of the mass redistribution is feasible.

There were changes in segmental inertial parameters due to muscular contraction. The center of mass moved higher up the shank, as more of the muscular mass moved in this direction. The moment of inertia about a given axis is a function of the distance in the other axis directions squared to each point mass comprising the body. The longitudinal moment of inertia increased slightly, as would be expected as muscle contraction preserves volume but increased the mean radius from this axis to the point masses. The transverse moments of inertia decreased slightly, as the mass was more compactly distributed about the center of mass in the contracted state.

The generation of muscular tension causes motion of the muscles; this study indicates that this results in a change in mass distribution. This highlights a weakness of the rigid body model commonly used in the analysis of human movement, as this redistribution of segmental mass may cause additional forces and moments to act at a joint. If during an impact the relative soft tissue motion is only as much as that which can be achieved voluntarily, although it is likely to be greater, this will 
lead to intra-segmental forces. A first estimate of these can be calculated assuming elastic deformation and solving for simple harmonic motion at a given frequency. This gives

$$
F=m \cdot(2 \pi f)^{2} \cdot x
$$

where

$$
\begin{aligned}
& \mathrm{F} \text { - force } \\
& \mathrm{m} \text { - mass } \\
& \mathrm{f} \text { - frequency of motion (in } \mathrm{Hz} \text { ) } \\
& \text { and } \mathrm{x} \text { is the displacement. }
\end{aligned}
$$

Fuller et al. (1997) showed a $14 \mathrm{~Hz}$ transient, in data from bone and soft tissue markers attached to the shank, during the heel impact during gait. Pain \& Challis (1998) have found similar frequency components for soft tissue motion of the thigh during impacts from landing from a drop. The wobbling mass is assumed to be $65 \%$ of the mass of the shank (Gruber et al., 1988). Therefore with a frequency of motion of $14 \mathrm{~Hz}$, a motion of $0.017 \mathrm{~m}$, and the soft tissue mass of a shank of 2.4 $\mathrm{kg}$, a maximal force of $315 \mathrm{~N}$ is obtained. This is not an insignificant force and one that would be ignored with the standard rigid body approach. The thigh would be expected to give even greater intrasegmental force values as it has a similar frequency response but a greater mass. This problem of nonrigidity is present in all body segments to some extent. Minetti \& Belli (1994) calculated a visceral mass of $8.8 \mathrm{~kg}$, with a displacement of at least $8 \mathrm{~cm}$ when oscillations were driven at a frequency of 3.5 $\mathrm{Hz}$. These values also lead to intra-segmental forces in the $300 \mathrm{~N}$ range.

Gruber et al. (1998) produced a computer simulation that showed a large decrease in hip and knee moments and constraining forces when a wobbling mass model was used over a rigid body model. In high frequency events such as impacts, information on the deformability of a segment will be necessary for a complete reconstruction of the forces acting throughout the system.

\section{REFERENCES}

Baca, A. (1996). Precise determination of anthropometric dimensions by means of image processing methods for estimating human body segment parameter values. Journal of Biomechanics, 29, 563-567.

Clauser, C.E., McConville, J.T., \& Young, J.W. (1969). Weight, volume and center of mass of segments of the human body. (AMRL Technical Report 69-70). Wright-Patterson Air Force Base, Ohio.

Crisco, J. J., \& McGovern, R. D. (1998). Efficient calculation of mass moments of inertia for segmented homogenous three-dimensional objects. Journal of Biomechanics, 31, 97-101.

Dempster, W. (1955). Space requirements of the seated operator. (WADC Technical Report 55-159). Wright-Patterson Air Force Base, Ohio.

Durkin, J., \& Dowling, J. (1998). A new technique for measuring body segment parameters using dual photon absorbtiometry. In Proceedings of $3^{\text {rd }}$ North American Congress on Biomechanics, August 14-18, University of Waterloo.

Fuller, J., Liu, L.-J., Murphy, M.C., \& Mann, R.W. (1997). A comparison of lower-extremity skeletal kinematics measured using skin- and pin-mounted markers. Human Movement Science, 16, 219242.

Gruber, K., Ruder, H., Denoth, J., \& Schneider, K. (1998). A comparative study of impact dynamics: wobbling mass model versus rigid body models. Journal of Biomechanics, 31, 439-444.

Hatze, H. (1980). A mathematical model for the computational determination of parameter values of anthropomorphic segments. Journal of Biomechanics, 13, 833-843.

Hinrichs, R.N. (1985). Regression equations to predict segmental moments of inertia from anthropometric measurements. Journal of Biomechanics, 18, 621-624. 
Jensen, R.K. (1978). Estimation of the biomechanical properties of three body types using a photogrammetric method. Journal of Biomechanics, 11, 349-358.

Minetti, A.E., \& Belli, G. (1994). A model for the estimation of visceral mass displacement in periodic movements. Journal of Biomechanics, 27, 1, 97-101.

Mungiole, M., \& Martin, P.E. (1990). Estimating segment inertial properties: Comparison of magnetic resonance imaging with existing methods. Journal of Biomechanics, 23, 1039-1046.

Pain, M.T.G., \& Challis, J.H. (1998). Measurement of the soft tissue motion of the thigh during an impact. In Proceedings of $3^{\text {rd }}$ North American Congress on Biomechanics, August 14-18, University of Waterloo.

Sarfaty, O., \& Ladin, Z. (1993). A video-based system for the estimation of the inertial properties of body segments. Journal of Biomechanics, 26, 1011-1016.

Stephenson, G. (1973). Mathematical Methods for Science Students. Longman Scientific and Technical, Harlow, Essex.

Yeadon, M.R. (1990). The simulation of aerial movement - Part II: A mathematical inertia model of the human body. Journal of Biomechanics, 23, 67-74. 